\title{
The discovery of the electromagnetic counterpart of GW170817: kilonova AT 2017gfo/DLT17ck
}

\author{
Stefano Valenti, ${ }^{1}$ David, J. Sand, ${ }^{2}$ Sheng Yang,,3 \\ and \\ Enrico Cappellaro, ${ }^{3}$ Leonardo Tartaglia, ${ }^{1,2}$ \\ and \\ Alessandra Corsi, ${ }^{4}$ Saurabh W. Jha, ${ }^{5}$ Daniel E. Reichart, ${ }^{6}$ Joshua Haislip, ${ }^{6}$ Vladimir \\ Kouprianov, ${ }^{6}$
}

Received __; accepted _

ApJL in press (DOI: 10.3847/2041-8213/aa8edf )

\footnotetext{
${ }^{1}$ Department of Physics, University of California, 1 Shields Avenue, Davis, CA 956165270, USA

${ }^{2}$ Department of Astronomy/Steward Observatory, 933 North Cherry Avenue, Room N204, Tucson, AZ 85721-0065, USA

${ }^{3}$ INAF Osservatorio Astronomico di Padova, Vicolo dellOsservatorio 5, I-35122 Padova, Italy

${ }^{4}$ Physics \& Astronomy Department, Texas Tech University, Lubbock, TX 79409, USA 0000-0003-3433-1492

${ }^{5}$ Department of Physics and Astronomy, Rutgers, TheState University of New Jersey, Piscataway, NJ 08854, USA

${ }^{6}$ Department of Physics and Astronomy, University of North Carolina at Chapel Hill, Chapel Hill, NC 27599, USA
} 


\begin{abstract}
During the second observing run of the Laser Interferometer gravitationalwave Observatory (LIGO) and Virgo Interferometer, a gravitational-wave signal consistent with a binary neutron star coalescence was detected on 2017 August 17th (GW170817), quickly followed by a coincident short gamma-ray burst trigger by the Fermi satellite. The Distance Less Than 40 (DLT40) Mpc supernova search performed pointed follow-up observations of a sample of galaxies regularly monitored by the survey which fell within the combined LIGO+Virgo localization region, and the larger Fermi gamma ray burst error box. Here we report the discovery of a new optical transient (DLT17ck, also known as SSS17a; it has also been registered as AT 2017gfo) spatially and temporally coincident with GW170817. The photometric and spectroscopic evolution of DLT17ck are unique, with an absolute peak magnitude of $M_{r}=-15.8 \pm 0.1$ and an $r$-band decline rate of $1.1 \mathrm{mag} / \mathrm{d}$. This fast evolution is generically consistent with kilonova models, which have been predicted as the optical counterpart to binary neutron star coalescences. Analysis of archival DLT40 data do not show any sign of transient activity at the location of DLT17ck down to $r \sim 19$ mag in the time period between 8 months and 21 days prior to GW170817. This discovery represents the beginning of a new era for multi-messenger astronomy opening a new path to study and understand binary neutron star coalescences, short gamma-ray bursts and their optical counterparts.
\end{abstract}

Subject headings: stars: neutron — surveys 


\section{Introduction}

The era of multi-messenger astronomy has truly begun. During the first Advanced LIGO (aLIGO, Aasi et al. 2015) run (O1), two definitive gravitational wave (GW) events were observed, corresponding to relatively massive black hole - black hole (BH-BH) mergers of 36+25 $M_{\odot}\left(\mathrm{GW} 150914\right.$; Abbott et al. 2016b) and 14+8 $M_{\odot}(\mathrm{GW} 151226$; Abbott et al. 2016a), respectively. These amazing discoveries were followed by a third event during the second aLIGO run (O2), which was another massive BH-BH merger (Abbott et al. 2017, 31+19 $\left.M_{\odot}\right)$. Each GW event was accompanied by a massive effort from the astronomical community to identify an electromagnetic (EM) counterpart (see e.g. Abbott et al. 2016), even though the likelihood of finding EM counterparts to $\mathrm{BH}-\mathrm{BH}$ mergers is low. On the other hand, GW events including at least one neutron star (NS; as either a NS-NS or NS-BH coalescence) are expected to produce a variety of EM signatures. Chief among them in the optical+near infrared regime is the so-called kilonova, resulting from the decay of r-process elements produced and ejected during the merger process (for a review, see Metzger 2017).

In order to search for kilonovae, two general approaches have been proposed: 1) wide-field searches of the aLIGO localization region (see e.g. Smartt et al. 2016) and 2) narrow-field targeted searches of galaxies both at the predicted GW event distance and within the sky localization region (e.g. Gehrels et al. 2016a). The $D<40$ (DLT40) Mpc one-day cadence supernova search uses the second approach, targeting galaxies within the GW localization region which are part of the main supernova search.

On 2017 August 17 (UT) a GW event was discovered by aLIGO and Virgo (Acernese et al. 2015) observatories (LIGO-Virgo collaboration, hereafter LVC) which was consistent with a neutron star binary coalescence with a low false alarm rate, at a distance of $D \sim 40 \mathrm{Mpc}$ (Goldstein et al. 2017, see Section 3 for further details). A potential short gamma-ray burst (GRB) counterpart was discovered by Fermi (GBM; trigger 
524666471). Soon after, multiple groups reported the detection of an optical counterpart (AT 2017gfo/DLT17ck/SSS17a; we will refer to the counterpart as DLT17ck throughout this work) which was subsequently identified as a true kilonova based on its fast spectroscopic (Lyman et al. 2017) and photometric (Yang et al. 2017a) evolution. The apparent host galaxy of DLT17ck is the normal early-type galaxy NGC 4993 (see Sadler et al. 2017).

In this paper we present the observations of the DLT40 team associated with the kilonova DLT17ck, based on our ongoing one-day cadence search. The DLT40 team was one of the initial groups reporting the discovery of the kilonova (Section 3), and based on our light curve and an early spectrum, we show that DLT17ck resembles the expected observables of a kilonova (Section 4). The DLT40 team also has a history of observations of the NGC 4993 field during the year before the GW event (Section 5). We end the paper by summarizing our results, and discussing prospects for electromagnetic counterpart searches with small telescopes (Section [6).

\section{DLT40 GW counterpart search}

The Distance Less than 40 Mpc survey (DLT40; Tartaglia et al., in preparation) is a one day cadence supernova search using a PROMPT $0.4 \mathrm{~m}$ telescope located at Cerro Tololo Inter-American Observatory (CTIO; Reichart et al. 2005). The survey goal is the early detection and characterization of nearby SNe. DLT40 has been operational since 2016, and observes 300-600 targeted galaxies on a nightly basis. A typical single-epoch integration of $45 \mathrm{~s}$ reaches a limiting magnitude of $r \approx 19 \mathrm{mag}$ with filterless observations. The field of view of the PROMPT camera is $10 \times 10 \mathrm{arcmin}^{2}$, sufficient to map all but the nearest galaxies in the search.

The DLT40 galaxy sample is drawn from the Gravitational Wave Galaxy Catalogue 
(GWGC; White et al. 2011), with further cuts made on recessional velocity $(V<3000$ $\mathrm{km} / \mathrm{s}$, corresponding to $D \lesssim 40 \mathrm{Mpc}$ ), declination (Dec $<+20 \mathrm{deg}$ ), absolute magnitude $\left(M_{B}<-18 \mathrm{mag}\right)$, and Milky Way extinction $\left(A_{V}<0.5 \mathrm{mag}\right)$. For these galaxies, we strive for a one-day cadence between observations to constrain the explosion epoch of any potential SN.

The DLT40 pipeline is totally automated, with pre-reduced images delivered from the telescope, ingested and processed in a few minutes. New transient candidates are detected on difference images and are available for visual inspection within $\sim 2-3$ minutes of ingestion. At the time of writing, DLT40 has discovered and confirmed 12 young SNe in the nearby Universe, with initial results reported in Hosseinzadeh et al. (2017).

The DLT40 GW follow-up strategy was planned as a straightforward addition to the core DLT40 supernova search. When a LVC gravitational wave event is announced, the BAYESTAR sky map (Singer \& Price 2016) is cross-matched with the DLT40 galaxy sample. All DLT40 galaxies that are within the LVC localization area are selected for high priority imaging in the nightly DLT40 schedule. Depending on the size of the LVC localization area and the number of galaxies selected, we apply a spatial cut (between the 80-99\% confidence localization contours of the LVC map) and/or a cut in luminosity to select the galaxies with most of the stellar mass. This strategy broadly follows that laid out by other LVC EM follow-up groups with narrow-field telescopes (e.g. Gehrels et al. 2016a). Further details of our search strategy, and our other results from O2, will be presented in a separate work (Yang et al. in preparation). 


\section{Discovery of DLT17ck}

On 2017 August 17.528 UT, the LVC reported the detection of a gravitational-wave nearly coincident in time (2 seconds before, Goldstein et al. 2017) with the Fermi GBM trigger $524666471 / 170817529$ located at $\mathrm{RA}=176.8^{\circ}$ and $\mathrm{DEC}=-39.8^{\circ}$ with an error of $11.6^{\circ}$ (at $1 \sigma$ ). The $\mathrm{LVC}$ candidate had an initial localization of $\mathrm{RA}=186.62^{\circ}, \mathrm{DEC}=-48.84^{\circ}$ and a $1 \sigma$ error radius of $17.45^{\circ}$ (LIGO/Virgo Collaboration 2017a). The GW candidate was consistent with a neutron star binary coalescence with false alarm rate of $\sim 1 / 10,000$ years (LIGO/Virgo Collaboration 2017a). The gravitational wave was clearly detected in the LIGO detectors but was below threshold for the Virgo detector (LIGO/Virgo Collaboration) 2017b). Despite this, the Virgo data were still crucial to further constrain the localization of the event to only $31 \mathrm{deg}^{2}$ (90\% credible region). The luminosity distance was constrained with LIGO data to be $40 \pm 8 \mathrm{Mpc}$ (LIGO/Virgo Collaboration 2017b). In Figure 11we show a map of both the LIGO+Virgo and Fermi GBM localizations, which overlapped on the sky. As part of the DLT40 search, we prioritized observations of 20 galaxies within the $99 \%$ confidence area of the LVC error-box and with a cut in luminosity. Among the 23 galaxies within the LIGO/Virgo error box, we selected the 20 galaxies within $99 \%$ of the cumulative luminosity distribution. At the same time, we also selected the 31 most luminous galaxies in the Fermi region of the coincident short GRB (see Figure 1). The 51 DLT40 galaxies selected were then observed at high priority. In this work, we present the only transient we detected within either the LVC or Fermi localizations: AT 2017gfo/DLT17ck (detected in in NGC 4993).

On 2017 August 17 23:49:55 UT (11.09 hours after the LVC event GW170817), we detected DLT17ck, at RA=13:09:48.09 and DEC=-23:22:53.4.6, 5.37W, 8.60S arcsec offset from the center of NGC 4993 (Yang et al. 2017b, see Figure 2). At the same time, DLT17ck was detected by Coulter et al. (2017), Allam et al. (2017), Melandri et al. (2017) and 
Arcavi et al. (2017a) and intensively observed by portions of the astronomical community that signed a memorandum of understanding with the LVC. DLT17ck was not reported to the internal (collaboration-wide) GCN by our team until a second confirmation image was obtained on August 18 00:40:38 UT. The LVC GW region of GW170817 was also observed in other windows of the EM spectrum, from radio to X-ray wavelengths. It was recovered in the UV, optical, and near infrared. Deep X-ray follow-up observations conducted with the Chandra observatory revealed X-ray emission from a point source at a position consistent with that of the optical transient DLT17ck (Bartos et al. 2017b; Fong et al. 2017; Troia et al. 2017). A radio source consistent with the position of DLT17ck (Adams et al. 2017) was detected with the Karl G. Jansky VLA (Mooley et al. 2017; Corsi et al. 2017), at two different frequencies $(\approx 3 \mathrm{GHz}$ and $\approx 6 \mathrm{GHz}$ ). Marginal evidence for radio excess emission at the location of DLT17ck was also found in ATCA images of the field at similar radio frequencies $(\approx 5 \mathrm{GHz}$; Bartos et al. 2017b). Finally, neutrino observations were reported with one neutrino detected within the preliminary LVC localization (Bartos et al. 2017a), though this was later established to be unrelated to GW170817/DLT17ck (Bartos et al. 2017b).

\section{DLT17ck: a new type of transient}

Our discovery magnitude $r=17.46 \pm 0.03 \mathrm{mag}$ at the distance of $39.5 \pm 2.6 \mathrm{Mpc}$ (distance modulus, $\mu=32.98 \pm 0.15$ mag using the Tully-Fisher relation Freedman et al. 2001) and Milky Way reddening $E(B-V)=0.109$ mag (Schlafly \& Finkbeiner 2011) brings DLT17ck to an absolute magnitude of $M_{r}=-15.8 \pm 0.1 \mathrm{mag}$. This magnitude is consistent with that typically observed in faint CC SNe (Spiro et al. 2014) and brighter than that of some kilonova models proposed so far. However, in the hours after the discovery, it became clear that DLT17ck was a unique event. DLT17ck was indeed cooling down and getting 
dimmer, much faster than any other SN we ever observed. About 35 hours after GW170817, DLT17ck had dimmed by almost a magnitude (Yang et al. 2017a). By day 5, DLT17ck was already $\sim 4$ magnitudes fainter than at discovery and disappeared below our magnitude limit the day after. At the same time, DLT17ck remained detectable in the near-infrared for a longer time. In Figure 3 (right panel), we compare the DLT40 light curve of DLT17ck with those of the most rapid transients available in the literature. DLT17ck evolves faster than any other known SN (gray points) and peaked probably between our discovery images and our third detection (respectively 11 and 35 hours after GW170817)1.

Regardless of the energy source powering them, the light curves of astronomical transients like supernovae and kilonovae are regulated by the same physics. At early times, the photons released can not immediately escape due to the high optical depth. The photon diffusion time depends on the ejecta mass, the opacity and the ejecta velocity (Arnett 1982). For kilonovae, the ejected mass has been predicted to be between $10^{-4}$ and $10^{-2}$ $\mathrm{M}_{\odot}$ depending on the lifetime of the hypermassive neutron star that forms at the moment of coalescence. A longer lifetime corresponds to a larger ejected mass and a brighter and longer-lasting optical electromagnetic counterpart (Kasen et al. 2013; Metzger 2017).

Because of the high neutron fraction the nucleosynthesis in the ejected material is driven by the r-process, producing a significant fraction of lanthanide that dominates the opacity. Because of a large uncertainty in lanthanide opacity, the ejecta opacity is not well constrained; it should be between 1 and $100 \mathrm{~cm}^{2} \mathrm{~g}^{-1}$ (closer to 1 for ejecta with a small amount of lanthanide elements; Metzgen 2017). Finally, velocities in the range 0.1-0.3 times the speed of light are also expected (see Metzger 2017, and reference therein). Using equation 5 from Metzger (2017),

\footnotetext{
${ }^{1}$ The possibility that DLT17ck is not related to GW170817, and exploded prior to the event, is discussed in Sec 5
} 


$$
\begin{array}{r}
t_{\text {peak }} \equiv\left(\frac{3 M k}{4 \pi \beta v c}\right)^{1 / 2} \approx 1.6 d\left(\frac{M}{10^{-2} M_{\odot}}\right)^{1 / 2}\left(\frac{v}{0.1 c}\right)^{-1 / 2} \\
\left(\frac{k}{1 c m^{2} g^{-1}}\right)^{1 / 2}
\end{array}
$$

where $\beta \approx 3, M$ is the ejected mass, $v$ is the expansion velocity, $k$ the opacity and $t_{\text {peak }}$ is the time of the peak, we can give a a rough estimate of the ejected mass. Soon after our first detection (11 hours after explosion), a few groups reported a flattening or slightly increase of the luminosity (Wolf et al. 2017; Arcavi et al. 2017a), but our second detection (35 hours after explosion) shows the object fading. We then assume Aug. 18.528 UT (24 hours after GW170817) as the epoch of the peak. We use an opacity of $1-10 \mathrm{~cm}^{2} / \mathrm{g}$ since the early blue peak should not contain large amounts of lanthanide (Metzger 2017) and an expansion velocity of $0.2 \times$ c. With these values, we obtain an ejected mass of $\approx 3 \times 10^{-3}$ $-10^{-2} \mathrm{M}_{\odot}$. However, the equation we used is an approximation and more careful models are needed. Comparing the DLT40 light curve with several kilonova models (see Figure 3), we found two models evolving as fast as DLT17ck which we describe below: The model by Metzger et al. 2010 (Met10) assumes a radioactive powered emission and an ejected mass of $10^{-2} \mathrm{M}_{\odot}$, outflow speed of $v=0.1 c$ and iron like opacity; the model by Barnes \& Kasen 2013 (B\&K) assumes an ejected mass of $10^{-3} \mathrm{M}_{\odot}$, velocity of $0.1 \mathrm{c}$ and a typical lanthanide opacity. Both models are consistent with the ejected mass we computed above, and support the kilonova interpretation.

Further evidence for the kilonova hypothesis comes from the analysis of DLT17ck spectra. Spectroscopic observations were performed by Shapee et al. (2017) about 12 hrs after GW170817, showing a blue and featureless continuum. This supports the idea that DLT17ck was discovered young, although a blue and featureless continuum is also common for young SNe II and GRB afterglows. The fast cooling of DLT17ck (and hence the small ejected mass) became evident as more spectra were collected. The extended-Public 
ESO Spectroscopic Survey for Transient Objects (ePESSTO Smartt et al. 2015) observed DLT17ck $~ 35$ hours after GW170817, reporting a featureless spectrum, with a much redder continuum than that observed in SN spectra at similar phases (Lyman et al. 2017, see Figure 4). A black-body fit to the spectrum revealed a temperature of $\approx 5200 \mathrm{~K}$. Considering a spherically symmetric explosion and a black body emission, the radius of the kilonova should have expanded from the radius of a neutron star (few tenth $10^{5} \mathrm{~cm}$ ) to $7.3 \times 10^{14} \mathrm{~cm}$. Under homologous expansion this requires a velocity expansion of $0.2 c$.

\section{Search for pre-discovery outbursts in historical data.}

In the standard kilonova model, we only expect a bright electromagnetic signature after coalescence. We can test this by looking at DLT40 observations taken before 2017 August 17. NGC 4993 is one of the galaxies monitored by the DLT40 supernova search, observed on average every 3 days from February 2017 to July 2017 (see Table 1). Our images show no sign of an optical transient down to a limit of $m_{r} \sim 19$ mag (see Figure 3), corresponding to $M_{r} \sim-14 \mathrm{mag}$ at the adopted distance of NGC 4993. Similarly, the field was also observed from 2013 to 2016 from La Silla QUEST on the ESO 1.0 meter telescope with no detection to a limit of $R \sim 18$ mag (Rabinowitz \& Baltay 2017).

The last DLT40 non-detection at the position of DLT17ck is on 2017 July 27th (21 days before the LVC event) down to $m_{r}=19.1 \mathrm{mag}$. Combining this limit with the extremely fast timescale of the transient, its blue continuum in the early spectra, its rapid cooling, and its photometric consistency with some kilonova models makes it extremely unlikely DLT17ck can be explained by any kind of supernova unrelated to the GW/GRB event. Rather, all the evidence favors that DLT17ck was discovered young, and is the optical counterpart of GW170817 and GRB 524666471/170817529. 


\section{Summary and Future Prospects}

In this paper, we presented the discovery of DLT17ck in the error region of the LVC event GW170817 and the Fermi short GRB 524666471/170817529. DLT17ck is characterized by a very fast optical evolution, consistent with some kilonova models and with a small ejecta mass $\left(10^{2}-10^{-3} \mathrm{M}_{\odot}\right)$. Spectroscopic observations conducted about 35 hrs after the explosion show a featureless continuum with blackbody temperature of 5200 K, confirming the fast evolution of DLT17ck compared to the evolution of other transients like classical supernovae. In addition, it is also surprising that at such a low temperature, no features are visible. We may speculate that this is the result of blending due to the high velocity of the expanding ejecta. Given the coincidence with the LVC event and the short Fermi GRB, it is likely the optical counterpart of the merging of two neutron stars in a binary system. This event represents a milestone for astronomy, being the first multimessenger event from which both photons and gravitational waves have been detected.

The unprecedented characteristics of DLT17ck raise a question as to the rates of such objects. The daily cadence of the DLT40 search can help constrain the rates of kilonovae and other rapidly-evolving transients. Details of rate measurements will be presented in a dedicated paper (Yang et al., in preparation), while here we report some of the results related to kilonovae. Using the galaxies within $40 \mathrm{Mpc}$ that we have observed in the last two years, and under the simplifying assumption that all kilonovae have a light curve similar to DLT17ck, we find an upper limit (at 95\% confident level) to the rate of kilonovae of $0.48_{-0.15}^{+0.9}$ binary neutron stars (BNS) SNu2 . For a Milky Way luminosity $\sim 2 \times 10^{10} L_{\odot}$, this translates to an upper limit of 9 Galactic kilonovae per millennium. This limit is not too stringent since it is two orders of magnitude larger than the Galactic rate of binary neutron star coalescence of $24 \mathrm{Myr}^{-1}$ estimated by Kim et al. (2015) from known neutron-star

$$
{ }^{2} \mathrm{SNu}=(100 y r)^{-1}\left(10^{10} L_{\odot}^{B}\right)^{-1}
$$


binaries.

We can convert our luminosity-based kilonova rate to a volumetric rate using the local luminosity density from Blanton et al. (2003). This gives a limit of $9.4 \pm 0.8 \times 10^{-5}$ kilonovae $\mathrm{Mpc}^{-3} \mathrm{yr}^{-1}$. This is consistent with previous limits $\left(<0.05 \mathrm{Mpc}^{-3} \mathrm{yr}^{-1}\right.$; Berger et al. 2013), that however were based on hypothetical parameters for the BNS optical light curve, and is comparable to the volumetric rate of fast optical transients, $4.8-8.0 \times 10^{-6} \mathrm{Mpc}^{-3}$ $\mathrm{yr}^{-1}$ found by Drout et al. (2014).

Looking forward to the O3 LVC run, in 2018, it is useful to explore strategies to detect EM counterparts of NS-NS mergers. DLT17ck was discovered independently by several groups (eg. SWOPE and DLT40; Coulter et al. 2017 and Yang et al. 2017b), using the approach of targeting nearby galaxies within the LVC region with small field-of-view instruments (Gehrels et al. 2016a). Several wide-field searches were also able to identify the transient (Allam et al. 2017; A. Mller 2017; Lipunov et al. 2017; Chambers et al. 2017), but only after reports from the targeted searches. This was likely due to the challenge of analyzing a large amount of data in a short period of time.

The small field-of-view strategy, and certainly our discovery, was successful because GW170817/DLT17ck was extremely nearby. The short Fermi GRB associated with DLT17ck is the closest ever discovered (see Berger 2014, for a review of short GRBs). However, with the expected increase in sensitivity of the LVC detectors, in O3 the volume where NS-NS mergers can be detected will reach $150 \mathrm{Mpc}$, increasing further to $200 \mathrm{Mpc}$ at full sensitivity (2019+; Abbott et al. 2016). At these distances galaxy catalogs are incomplete (Smartt et al. 2016) and the sheer number of galaxies will likely favor wide-field strategies. Nonetheless, because the Virgo horizon distance during O3 is predicted to be $65-115 \mathrm{Mpc}$ (Abbott et al. 2016), the small field-of-view strategy may still be important for the best-localized sources. DLT40 reaches a limiting magnitude of $r \sim 19$ mag in 45 to 
60 second exposures. Taking a more conservative limit of $18.5 \mathrm{mag}$, we would expect to be able to see sources like DLT17ck out to $70 \mathrm{Mpc}$. Increasing the exposure time to reach a depth of $\sim 20$ mag would allow us to observe binary neutron star mergers in the full range of the Virgo interferometer.

Research by DJS and L.T. 1 is supported by NSF grant AST-1412504 and AST-1517649. AC acknowledges support from the NSF award \#1455090. The work of SY was supported by the China Scholarship Council(NO. 201506040044). 


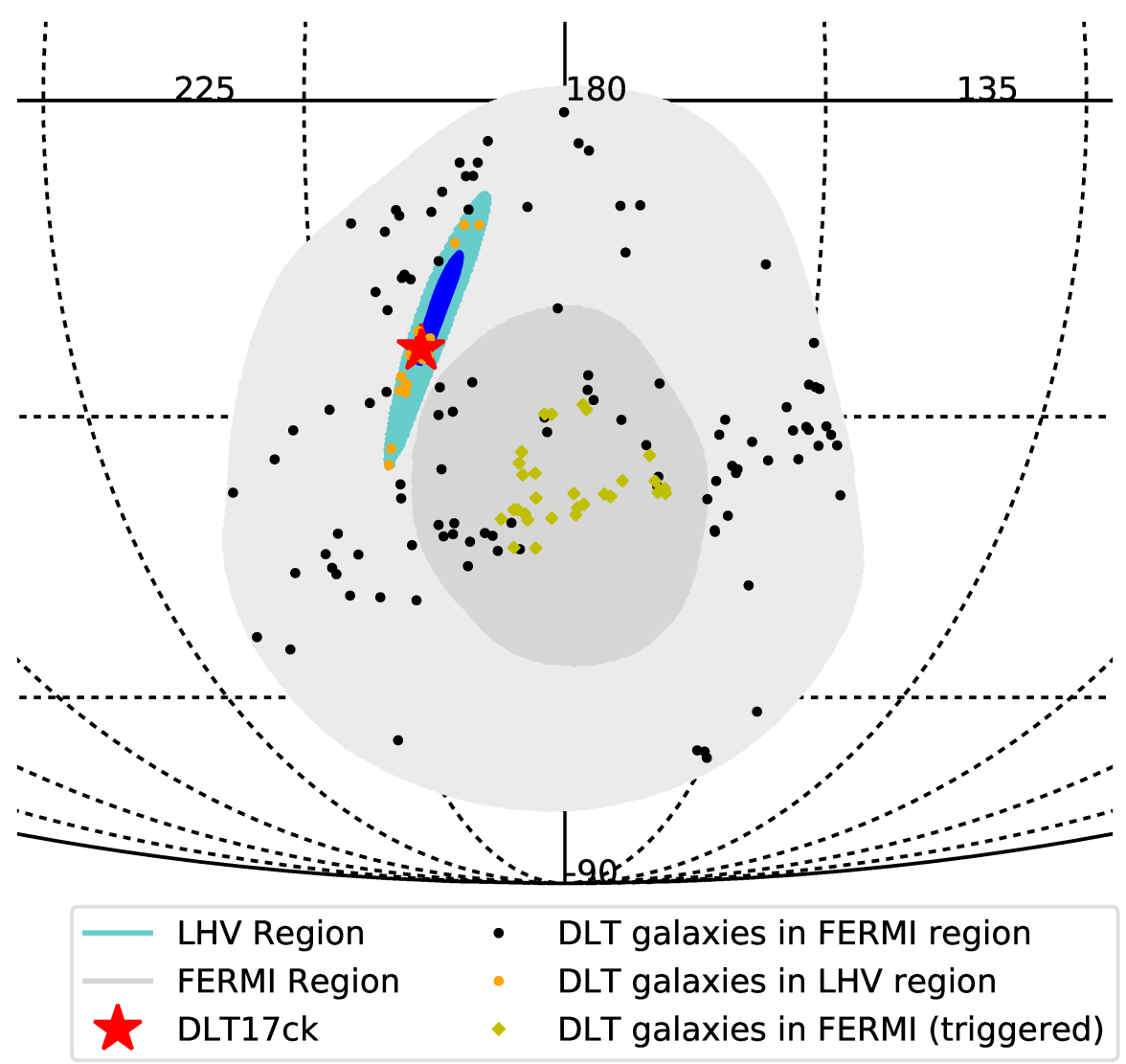

Fig. 1. - The sky map region of the GW170817 LVC event using all three gravitationalwave observatories (H1, L1, and V1) over-imposed on the Fermi localization of GBM trigger 524666471/170817529. The DLT40 galaxies observed the first Chilean night after the LVC trigger are marked in orange (galaxies within the LVC region) and in olive green (galaxies within the Fermi localization). The remaining black points are those DLT40 galaxies which were within the Fermi localization but were not observed by our program. The red star marks the location of DLT17ck and the host galaxy NGC 4993. 
Table 1: Photometric Data for DLT17ck

\begin{tabular}{|c|c|c|c|c|c|c|c|c|c|}
\hline Date & JD & $\operatorname{mag}^{a b}$ & Filter $^{c}$ & telescope & Date & JD & $\operatorname{mag}^{a b}$ & Filter $^{c}$ & telescope \\
\hline 2017-08-17 & 2457983.493 & 17.460 .03 & $r$ & Prompt 5 & 2017-03-07 & 2457819.772 & $>20.90$ & $r$ & Prompt 5 \\
\hline 2017-08-18 & 2457983.528 & 17.560 .04 & $r$ & Prompt 5 & 2017-03-10 & 2457822.595 & $>19.97$ & $r$ & Prompt 5 \\
\hline 2017-08-18 & 2457984.491 & 18.000 .06 & $r$ & Prompt 5 & 2017-03-11 & 2457823.592 & $>19.37$ & $r$ & Prompt 5 \\
\hline 2017-08-19 & 2457984.510 & 18.290 .06 & $r$ & Prompt 5 & 2017-03-12 & 2457824.594 & $>19.39$ & $r$ & Prompt 5 \\
\hline 2017-08-19 & 2457985.476 & 19.340 .08 & $r$ & Prompt 5 & 2017-03-13 & 2457825.586 & $>19.20$ & $r$ & Prompt 5 \\
\hline 2017-08-19 & 2457985.478 & 19.290 .12 & $r$ & Prompt 5 & 2017-03-26 & 2457838.881 & $>20.37$ & $r$ & Prompt 5 \\
\hline 2017-08-21 & 2457986.503 & 20.180 .10 & $r$ & Prompt 5 & 2017-03-27 & 2457839.714 & $>21.14$ & $r$ & Prompt 5 \\
\hline 2017-08-22 & 2457987.504 & 20.920 .12 & $r$ & Prompt 5 & $2017-03-28$ & 2457840.717 & $>20.86$ & $r$ & Prompt 5 \\
\hline $2017-07-27$ & 2457961.599 & $>19.84$ & $r$ & Prompt 5 & 2017-03-29 & 2457841.720 & $>21.03$ & $r$ & Prompt 5 \\
\hline $2017-07-27$ & 2457962.495 & $>19.36$ & $r$ & Prompt 5 & 2017-03-30 & 2457842.666 & $>20.74$ & $r$ & Prompt 5 \\
\hline 2017-05-15 & 2457888.762 & $>19.79$ & $r$ & Prompt 5 & 2017-03-31 & 2457843.713 & $>20.83$ & $r$ & Prompt 5 \\
\hline 2017-05-16 & 2457889.751 & $>19.88$ & $r$ & Prompt 5 & 2017-04-02 & 2457845.704 & $>20.90$ & $r$ & Prompt 5 \\
\hline 2017-05-17 & 2457890.796 & $>19.61$ & $r$ & Prompt 5 & 2017-04-04 & 2457847.695 & $>20.73$ & $r$ & Prompt 5 \\
\hline $2017-05-20$ & 2457893.500 & $>19.88$ & $r$ & Prompt 5 & 2017-04-05 & 2457848.700 & $>20.87$ & $r$ & Prompt 5 \\
\hline 2017-05-21 & 2457894.562 & $>20.27$ & $r$ & Prompt 5 & 2017-04-06 & 2457849.857 & $>20.63$ & $r$ & Prompt 5 \\
\hline 2017-05-22 & 2457895.545 & $>20.65$ & $r$ & Prompt 5 & 2017-04-07 & 2457850.699 & $>20.24$ & $r$ & Prompt 5 \\
\hline $2017-05-28$ & 2457901.715 & $>19.69$ & $r$ & Prompt 5 & 2017-04-08 & 2457851.695 & $>19.74$ & $r$ & Prompt 5 \\
\hline 2017-05-29 & 2457902.548 & $>20.16$ & $r$ & Prompt 5 & 2017-04-09 & 2457852.679 & $>19.56$ & $r$ & Prompt 5 \\
\hline 2017-05-30 & 2457903.547 & $>20.12$ & $r$ & Prompt 5 & 2017-04-13 & 2457856.861 & $>19.45$ & $r$ & Prompt 5 \\
\hline 2017-05-31 & 2457904.544 & $>20.70$ & $r$ & Prompt 5 & 2017-04-14 & 2457857.678 & $>18.41$ & $r$ & Prompt 5 \\
\hline 2017-06-01 & 2457905.542 & $>20.45$ & $r$ & Prompt 5 & 2017-04-15 & 2457858.666 & $>20.07$ & $r$ & Prompt 5 \\
\hline 2017-06-02 & 2457906.511 & $>20.24$ & $r$ & Prompt 5 & 2017-04-21 & 2457864.654 & $>21.01$ & $r$ & Prompt 5 \\
\hline 2017-06-02 & 2457907.498 & $>20.06$ & $r$ & Prompt 5 & 2017-04-22 & 2457865.642 & $>20.93$ & $r$ & Prompt 5 \\
\hline 2017-06-19 & 2457923.645 & $>20.61$ & $r$ & Prompt 5 & 2017-04-23 & 2457866.760 & $>21.02$ & $r$ & Prompt 5 \\
\hline 2017-06-19 & 2457924.481 & $>19.65$ & $r$ & Prompt 5 & $2017-04-24$ & 2457867.652 & $>21.04$ & $r$ & Prompt 5 \\
\hline 2016-12-21 & 2457743.834 & $>20.44$ & $r$ & Prompt 5 & 2017-04-26 & 2457869.631 & $>20.67$ & $r$ & Prompt 5 \\
\hline 2017-02-06 & 2457790.858 & $>21.39$ & $r$ & Prompt 5 & 2017-04-27 & 2457870.626 & $>20.92$ & $r$ & Prompt 5 \\
\hline $2017-02-07$ & 2457791.823 & $>21.34$ & $r$ & Prompt 5 & $2017-04-28$ & 2457871.622 & $>20.65$ & $r$ & Prompt 5 \\
\hline $2017-02-08$ & 2457792.826 & $>21.26$ & $r$ & Prompt 5 & 2017-04-29 & 2457872.694 & $>20.66$ & $r$ & Prompt 5 \\
\hline 2017-02-09 & 2457793.835 & $>21.10$ & $r$ & Prompt 5 & 2017-04-30 & 2457873.618 & $>20.71$ & $r$ & Prompt 5 \\
\hline $2017-02-10$ & 2457794.824 & $>20.58$ & $r$ & Prompt 5 & 2017-05-01 & 2457874.615 & $>20.60$ & $r$ & Prompt 5 \\
\hline 2017-02-11 & 2457795.825 & $>20.33$ & $r$ & Prompt 5 & 2017-05-03 & 2457876.665 & $>20.76$ & $r$ & Prompt 5 \\
\hline 2017-02-12 & 2457796.756 & $>19.90$ & $r$ & Prompt 5 & 2017-05-04 & 2457877.594 & $>20.55$ & $r$ & Prompt 5 \\
\hline $2017-02-13$ & 2457797.747 & $>20.16$ & $r$ & Prompt 5 & 2017-05-05 & 2457878.606 & $>20.30$ & $r$ & Prompt 5 \\
\hline $2017-02-14$ & 2457798.692 & $>19.85$ & $r$ & Prompt 5 & 2017-05-06 & 2457879.577 & $>20.25$ & $r$ & Prompt 5 \\
\hline 2017-02-17 & 2457801.725 & $>20.37$ & $r$ & Prompt 5 & $2017-08-25$ & 2457990.504 & $>20.89$ & $r$ & Prompt 5 \\
\hline 2017-02-19 & 2457803.828 & $>20.83$ & $r$ & Prompt 5 & $2017-08-26$ & 2457991.504 & $>20.37$ & $r$ & Prompt 5 \\
\hline 2017-03-05 & 2457817.886 & $>20.78$ & $r$ & Prompt 5 & $2017-08-26$ & 2457992.489 & $>19.90$ & $r$ & Prompt 5 \\
\hline 2017-03-06 & 2457818.784 & $>20.91$ & $r$ & Prompt 5 & - & - & - & - & - \\
\hline
\end{tabular}

(a) : Data has not been corrected for extinction.

(b) : Limit magnitude are $5 \sigma$ detection limit.

(c) : Open filter calibrated to $r$. 


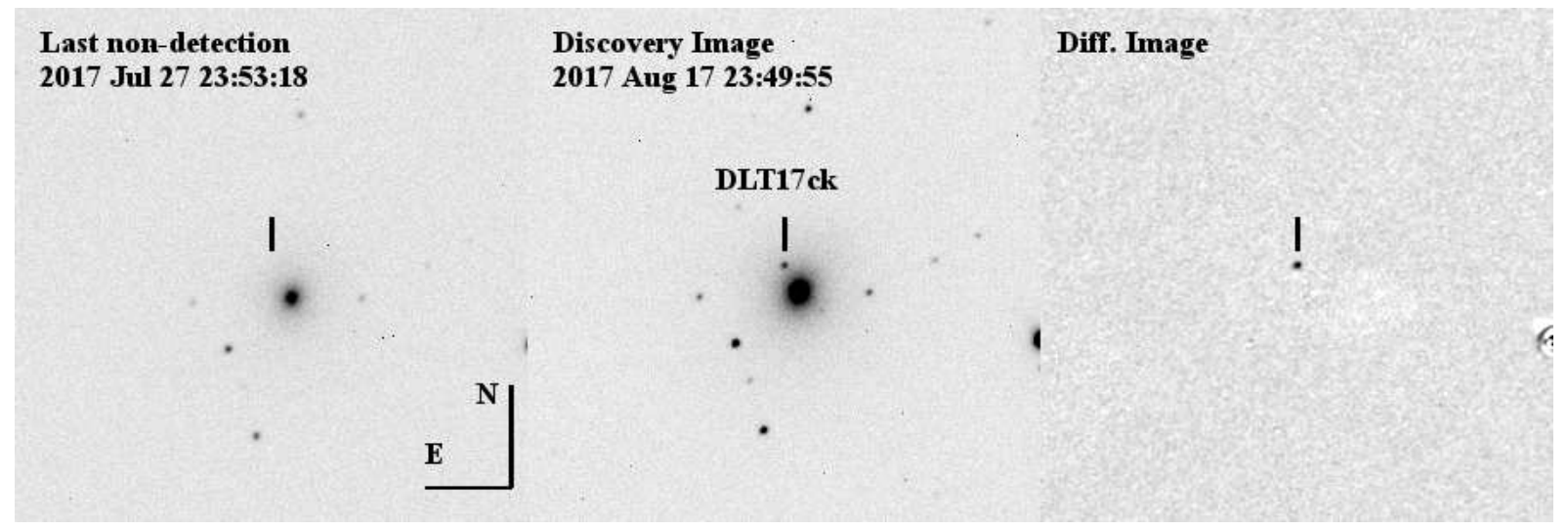

Fig. 2.- Last non-detection (on the left), discovery image of DLT17ck observed on 201708-17 at 23:49:55 UT. The difference image is shown on the right, where DLT17ck is clearly visible. 


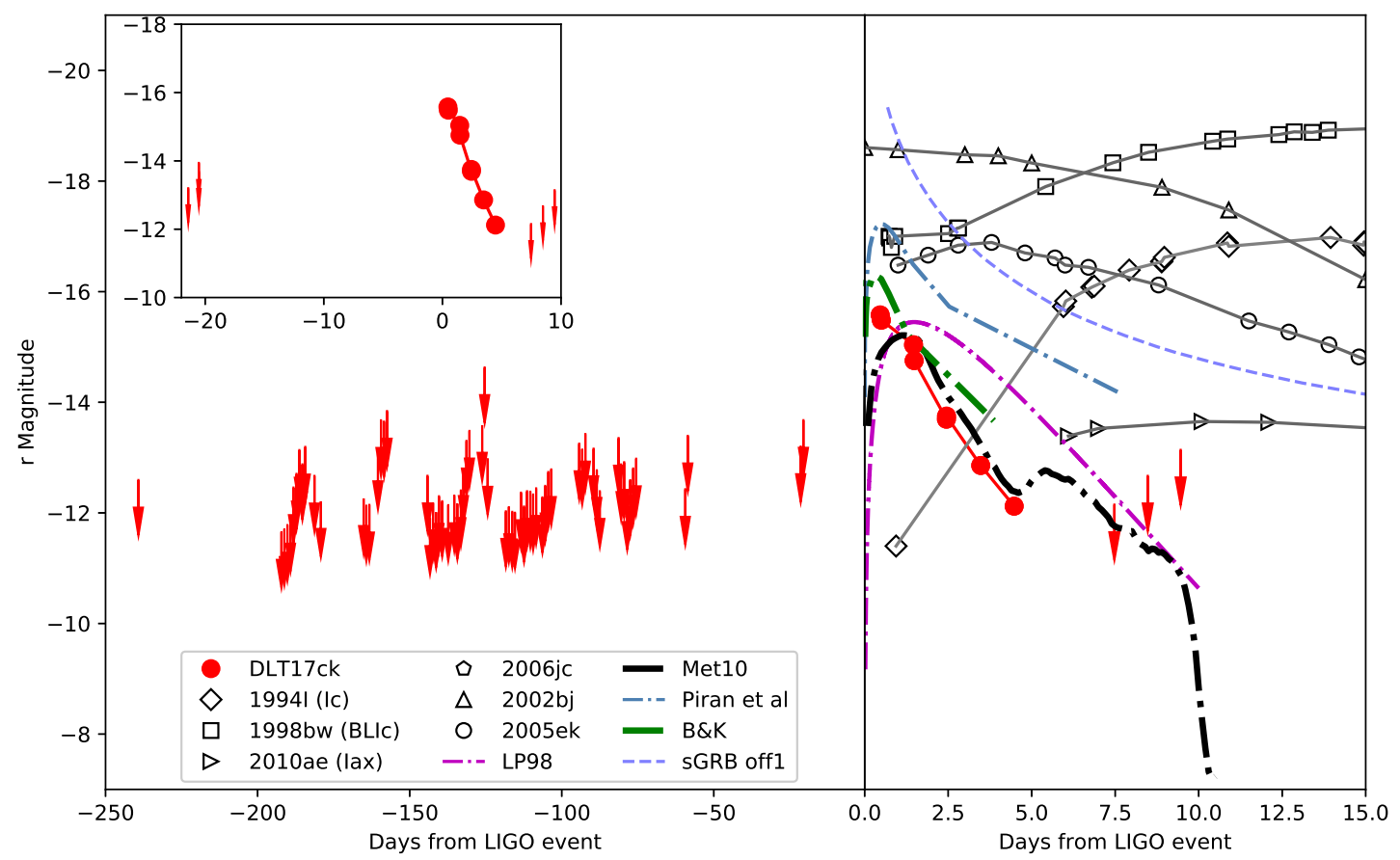

Fig. 3.- Right panel: DLT40 light curve of DLT17ck (in red) over plotted with normal or fast-evolving SNe (in gray). Several NS-NS merger models, scaled to a distance of $40 \mathrm{Mpc}$, are shown as comparison from Li \& Paczyński 1998 [LP98]; Metzger et al. 2010 [Met10]; Barnes \& Kasen 2013 [B\&K] and Piran et al. 2013 [Piran et al]. Left panel: We show the detection limits in the position of DLT17ck in the 6 months before GW170817 and an inset with the detected light curve 


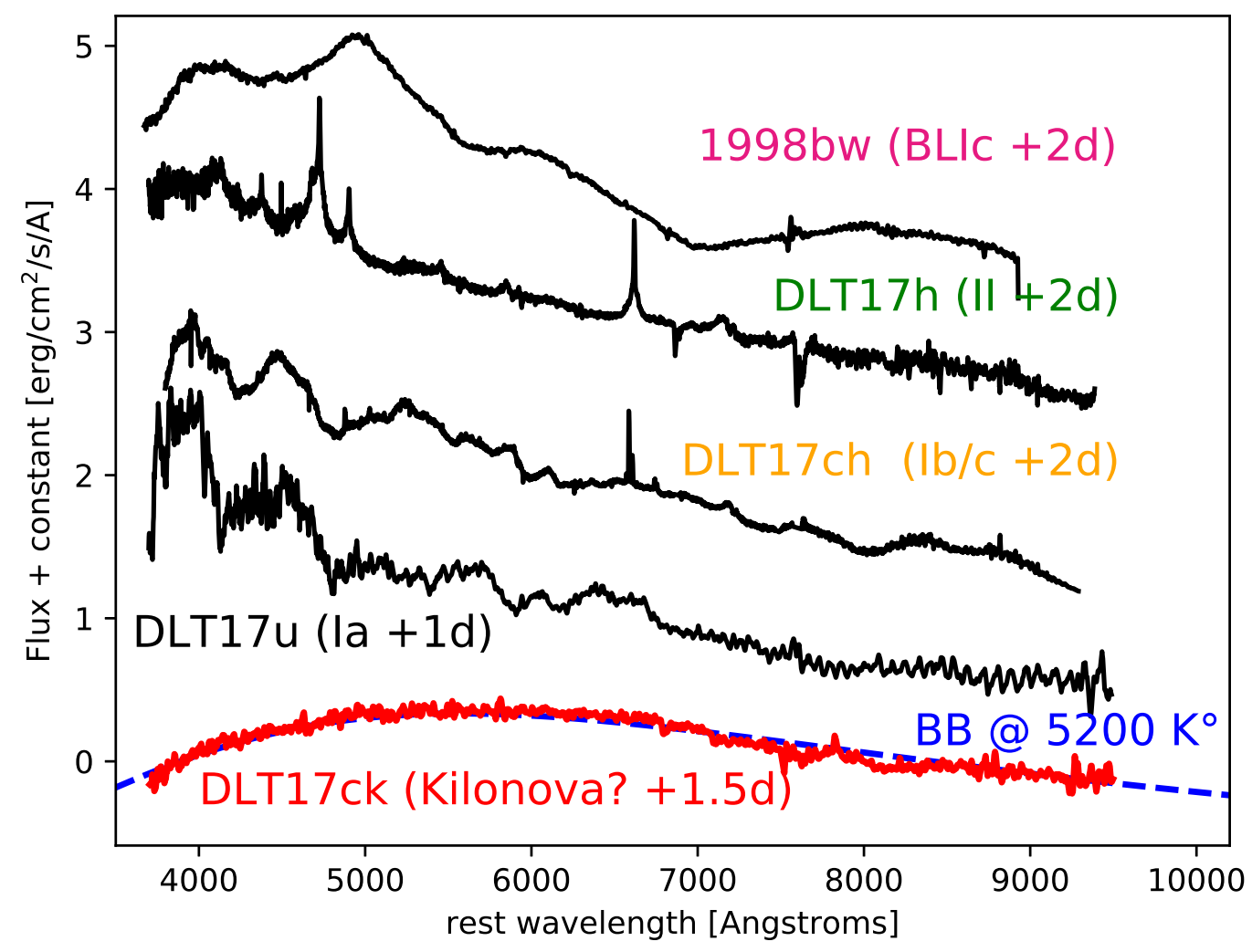

Fig. 4.- DLT17ck spectrum at 35 hours after the GW170817 compared with spectra of young SNe at similar epochs. DLT17ck is cooling much faster than any previously observed explosive transient. A blackbody fit indicates a temperature of $\approx 5200 \mathrm{~K}$. Data from: DLT17u (FLOYDS), DLT17ch (SALT), DLT17h (SALT), DLT17ck (NTT), SN1998bw (Danish 1.54 telescope + DFOSC). The presence of an emission feature at $\sim 7800 \AA$ is suspicious due to the presence of telluric lines close its position. 


\section{REFERENCES}

A. Mller, S. Chang, C. W. 2017, GRB Coordinates Network, 21542

Aasi, J., Abadie, J., Abbott, B. P.,et al. 2015, Classical and Quantum Gravity, 32, 115012

Abbott, B. P., Abbott, R., Abbott, T. D., et al. 2016a, Phys. Rev. Lett., 116, 241103

Abbott, B. P., Abbott, R., Abbott, T. D., et al. 2016, ApJ, 826, L13

Abbott, B. P., Abbott, R., Abbott, T. D., et al. 2016b, Phys. Rev. Lett., 116, 061102

Abbott, B. P., Abbott, R., Abbott, T. D., et al. 2016, Living Reviews in Relativity, 19, 1

Abbott, B. P., Abbott, R., Abbott, T. D., et al. 2017, Phys. Rev. Lett., 118, 221101

Acernese, F., Agathos, M., Agatsuma, K., et al. 2015, Classical and Quantum Gravity, 32, 024001

Adams, S. M., Kasliwal, M. M., \& Blagorodnova, N. 2017, LIGO-Virgo GRB Coordinate Network Circular, 21816

Allam, S., Annis, J., Berger, E., et al. 2017, GRB Coordinates Network, 21530

Arcavi, I., Howell, D. A., McCully, C., et al. 2017, Nature in press (DOI: 10.1038/nature24291)

Arnett, W. D. 1982, ApJ, 253, 785

Barnes, J., \& Kasen, D. 2013, ApJ, 775, 18

Bartos, I., Countryman, S., Finley, C., et al. 2017a, GRB Coordinates Network, 21511

Bartos, I., Countryman, S., Finley, C., et al. 2017b, GRB Coordinates Network, 21568

Berger, E. 2014, ARA\&A, 52, 43 
Berger, E., Leibler, C. N., Chornock, R., et al. 2013, ApJ, 779, 18

Blanton, M. R., Hogg, D. W., Bahcall, N. A., et al. 2003, ApJ, 592, 819

Chambers, K. C., Huber, M. E., Smartt, S. J., et al. 2017, GRB Coordinates Network, 21553

Corsi, A., Hallinan, G., Mooley, K., et al. 2017, LIGO-Virgo GRB Coordinate Network Circular, 21815

Coulter, D. A., Kilpatrick, C. D., Siebert, M. R., et al. 2017, Science in press (DOI:10.1126/science.aap9811)

Drout, M. R., Chornock, R., Soderberg, A. M., et al. 2014, ApJ, 794, 23

Shapee B. J., et al. 2017, Science in press (DOI: 10.1126/science.aaq0186)

Fong, W., Margutti, R., \& Haggard, D. 2017, LIGO-Virgo GRB Coordinate Network Circular, 21786

Freedman, W. L., Madore, B. F., Gibson, B. K., et al. 2001, ApJ, 553, 47

Gehrels, N., Cannizzo, J. K., Kanner, J., et al. 2016a, ApJ, 820, 136

Goldstein, A., et al. 2017, ApJL in press (DOI: 10.3847/2041-8213/aa8f41)

Hosseinzadeh, G., Sand, D. J., Valenti, S., et al. 2017, ApJ, 845, L11

Kasen, D., Badnell, N. R., \& Barnes, J. 2013, ApJ, 774, 25

Kim, C., Perera, B. B. P., \& McLaughlin, M. A. 2015, MNRAS, 448, 928

Li, L.-X., \& Paczyński, B. 1998, ApJ, 507, L59

LIGO/Virgo Collaboration. 2017a, GRB Coordinates Network, 21505 
LIGO/Virgo Collaboration. 2017b, GRB Coordinates Network, 21513

Lipunov, V., Gorbovskoy, E., V.G.Kornilov, et al. 2017, GRB Coordinates Network, 21546

Lyman, J., Homan, D., Maguire, K., et al. 2017, GRB Coordinates Network, 21582

Melandri, A., Campana, S., Covino, S., et al. 2017, GRB Coordinates Network, 21532

Metzger, B. D. 2017, Living Reviews in Relativity, 20, 3

Metzger, B. D., Martínez-Pinedo, G., Darbha, S., et al. 2010, MNRAS, 406, 2650

Mooley, K., Hallinan, G., \& Corsi, A. 2017, LIGO-Virgo GRB Coordinate Network Circular, 21814

Piran, T., Nakar, E., \& Rosswog, S. 2013, MNRAS, 430, 2121

Rabinowitz, D., \& Baltay, C. 2017, GRB Coordinates Network, 21599

Reichart, D., Nysewander, M., Moran, J., et al. 2005, Nuovo Cimento C Geophysics Space Physics C, 28, 767

Sadler, E. M., Allison, J. R., Kaplan, D. L., Murphy, T., \& on behalf of the VAST collaboration. 2017, GRB Coordinates Network, 21645

Schlafly, E. F., \& Finkbeiner, D. P. 2011, ApJ, 737, 103

Singer, L. P., \& Price, L. R. 2016, Phys. Rev. D, 93, 024013

Smartt, S. J., Valenti, S., Fraser, M., et al. 2015, A\&A, 579, A40

Smartt, S. J., Chambers, K. C., Smith, K. W., et al. 2016, ApJ, 827, L40

Spiro, S., Pastorello, A., Pumo, M. L., et al. 2014, MNRAS, 439, 2873 
Troja, E., Piro, L., van Eerten, H. J, \& Lien, A. 2017, Nature in press (DOI: $10.1038 /$ nature24290)

White, D. J., Daw, E. J., \& Dhillon, V. S. 2011, Classical and Quantum Gravity, 28, 085016

Wolf, C., Chang, S., \& Muller, A. 2017, GRB Coordinates Network, 21560

Yang, S., Valenti, S., Sand, D., et al. 2017a, GRB Coordinates Network, 21579

Yang, S., Valenti, S., Sand, D., et al. 2017b, GRB Coordinates Network, 21531

This manuscript was prepared with the AAS IATEX macros v5.2. 